\title{
Internet-based focus groups: New approaches to an 'old' research method
}

\section{Sarah Collard ${ }^{1}$, Edwin van Teijlingen $^{2,3,4^{*}}$}

${ }^{1}$ Post-doctoral researcher, Faculty of Health \& Social Sciences, Bournemouth University, Bournemouth, UK ${ }^{2}$ Professor of Maternal \& Perinatal Health Research, Faculty of Health \& Social Sciences, Bournemouth University, Bournemouth, UK ${ }^{3}$ Visiting Professor, Manmohan Memorial Institute of Health Sciences, Nepal ${ }^{4}$ Visiting Professor, Nobel College, Nepal

\section{Received:}

14 May 2016

Revised:

11 July 2016

Accepted:

10 August 2016

\section{${ }^{*}$ Correspondence: evteijlingen@bourne- mouth.ac.uk \\ Centre for Midwifery, Ma- ternal \& Perinatal Health Faculty of Health \& Social Sciences \\ Bournemouth House 19, Christchurch Road Bournemouth University Bournemouth BU13LH England, UK}

\begin{abstract}
Focus group (FG) discussions are a popular qualitative research method used in health care and health service research. More recently, internet-based FGs have grown in popularity due to the growth of: (a) the internet, both in terms of technical capacity and number of users; and (b) the improved quality of communication software (e.g. Skype). This paper highlights some of the strengths and weaknesses of conducting FGs online. Building on our experience of conducting traditional and internet-based FGs, we offer some practical advice to potential users.
\end{abstract}

\section{Background}

There is extensive research methods literature on conducting focus groups (FGs) in the field of health care and health service research (1-5), including in this journal, that recently published a paper on conducting FGs research in Nepal (6). This previous article has largely focused on traditional in-person FGs which consist of bringing participants together in a particular physical location at a given time to discuss issues in person. There are several limitations to running these traditional FGs. First, they rely on multiple individuals coming together at the same place, which can be problematic. Secondly, the ability to secure confidentiality outside the group is a concern. Finally, there are multiple expenses associated with organising and conducting traditional in-person FGs (e.g., travel expenses, catering and room hire, transcription) (7). However, with the growing technological options being made available through the internet, and the growth in internet users as well as the improved quality of communication programmes, it has now become more common to conduct internet-based FGs.

This paper highlights the two major variants of internet-based FGs: (a) written chat; and (b) audio/ video conferencing. We highlight the differences and similarities of these two approaches, the strengths and limitations of each approach and finish with advice for researchers beginning to employ internet-based FG methods. The first approach to be highlighted is that of online written FGs, which is the older of the two internet-based FG approaches.

\section{Online written focus groups}

Online written FGs use 'chat room' based technology in order to create a discussion on a common topic (see Table 1). These have shown to be cost-effective as they do not cost as much to run as in-person FGs, provide enhanced confidentiality as participants' names and faces can be kept anonymous from the group, and allow for more sensitive topics to be discussed in-depth and over time (7). Conducted as a chat room, the participants are provided with confidential pseudonyms to protect identity and asked to discuss the mutual topic. Using a chat room approach, this internet-based FG generates a real-time written record of the conversation which serves as the transcript for qualitative analysis and thereby reducing overall research costs. Along with these benefits, the use of such technology provides more succinct responses of the participants, rather than longer and sometimes off-topic responses that can result as part of an in-person face-to-face FG (7). Unlike in-person or video FGs, this style also allows participants to discuss a topic over time. If desired, researchers can set up the FG to run over days or even a few weeks, allowing participants to reply to previous entries which may have been made days or weeks earlier. This is referred to as asynchronous communication (8), as the discussion is not in real time but participants may 'drop-in' anytime they are able in order to reply to previous comments and observations. This allows for constant debate among participants as well as creates a dynamic debate that can shift and change backward and forward a few times.

Although there are many benefits of using written FGs, there are also drawbacks. For instance, some styles allow participants to chat at the same time, thus chatting over each other, creating an environment that does not allow a debate to flow as it would in a face-to-face session (7). As this is a more staged environment, the connection between the participants can create a stale atmosphere and lacks emotion compared to in-person and even video FGs. In addition, as the participant's response is only through text, voice connotations, expressions and body language cannot be heard or seen, thus text could be interpreted in a way 
that it was not intended to. Table 2 presents the strengths and weaknesses of this written style.

\section{Table 1 Example of online written focus group (on maternal health \& development)}

Facilitator: Only people signed up to this internet-based Focus group discussion can add comments. [This comment should help assure your FG participants that only authorised participants will take part and that the risk of unauthorised access is minimalized. The transcript below the names made up for this example are changed into 'participant $\mathrm{x}$ ' ]

April 19, 2016 13:44

Participant 1: the MDGs have made very little difference to childhood mortality in Nepal but most certainly a lot to maternal mortality. It is often hailed as one of the successes of health policy and interventions in our country.

April 19, 2016 13:48

Participant 2: I really liked the emphasis on maternal health improvement in MDG5.

April 19, 2016 15:37

Facilitator: Does everybody know that MDG stands for 'Millennium Development Goals'? Also just in case you didn't know MDG5 is the fifth Millennium Development Goals and focuses on improving maternal mortality.

April 20, 2016 00:22

Participant 3: Nepal did better in MDG5 than many other south Asian countries

April 20, 2016 03:11

Participant 2: I found this link to a paper in Health Prospect on the web you might like to read [Participant 1]: http://www.nepjol. info/index.php/HPROSPECT/issue/view/986

April 20, 2016 04:27

Participant 1: Thanks will read.

April 20, 2016 06:52

Participant 3: MDGs finished in 2015, pity really.

April 20, 2016 11:51

Participant 3: Sorry forgot to mention maternal health improved after introduction of abortion legislation in Nepal.

April 20, 2016 14:22

Participant 1: Just read [Participant's 2] suggested piece online. Where next with Sustainable Development Goals?

April 20, 2016 15:16

Facilitator: Interesting [Participant 1] mentions Sustainable Development Goals (SDGs) as successors of MDGs. What do people in the Focus Group think of the new SDGs?

April 20, 2016 15:27

Participant 3: There is one SDG out of 17 focused on health.

April 20, 2016 16:42

Participant 4: MDGs had three health goals out of total of eight, so fewer in SDGs

April 20, 2016 17:45

Participant 3: SDGs more on general development less on health!!!

.........etc.

Table 2 Strengths and weaknesses of online written FG

\section{Strengths}

- Global participation

- Cheap

- Run more FGs same time

- Creates instant written record

- Not necessarily in real time

- Facilitator asks follow-up questions, show photos, videos, etc.

to stimulate discussion.

- Facilitator can do interim analysis as transcript is generated as online chat continues and hence raise specific questions.

- Facilitators can send email updates to keep participants interested /adding text.

\section{Weaknesses}

- Typing text is slower than talking

- It can limit expression, i.e. more artificial than speaking one's mind and people may self-sensor during or immediately after having written comments.

- Different people participate at different times (perhaps days apart) in different moods and perhaps having been influences about the FG topic by the media or friends.

- Others (participants) may make a record of FG without the facilitator or fellow participants knowing this.

\section{Online audio/video discussions}

More recently the growth of internet capacity and programmes such as Skype, GoToMeeting, and BlueJeans have made it possible to conduct FGs online using video conferencing technology. These 'live' FGs are synchronous or in real-time (8). These are similar to telephone-based focus groups in that participants do not have to be in the same room to take part, which suits research covering a large geographical area and/or people with very busy schedules (9). This approach has some major benefits as there is no longer a 
need for participants to meet in-person, thus reducing travel time whilst still enabling them to communicate freely in a 'real' conversation with others. This enables participants to react to each other's comments, questions, and observations. Also, viewing each other as separate windows on the screen seems to create a group environment and encourages participants to talk more to each other. The domination of one individual could still result, however, the discussion can often go in a round and each person takes a turn to speak. This differs compared to traditional in-person FGs where there can be two or three people dominating the conversation. Like a traditional in-person FG, this type of internet-based FG will have a facilitator and can be recorded. This should give the researcher a rich data set as one would expect to generate from a good qualitative study.

As with all methods, internet-based FGs also have a few potential limitations such as: privacy, a lack of ability to read body language, and technological issues (10). In regards to privacy, if your participant is joining in from home or a busy office, there might be a lack of privacy. Researchers' colleagues, partners or children may come into view, or worse, join in the discussion with your participants in the internet-based FG. Reading participants' body language in an in-person FG allows for the researcher to gain access to undisclosed feelings as a result of participants sitting, for example, cross-armed or open. Within internet-based FGs, the individual does not always display the same body language as other participants are not in the room with them and as a result, he/she may not respond in the same physical way. In addition, web-cameras usually only present the upper torso or head of the individual, making the researcher unable to read the participant's body language. Finally, technological issues pose a difficulty as internet connections can drop suddenly, be slow or 'patchy' for certain individuals, and can pose problems for those who may not be used to using a certain new type of technology (10). In addition, some participants might not like to use video and only use audio communication channels. For example, they may switch off the camera whilst using Skype. This results in a FG where you can see some but not all, making communication between participants slightly unequal.

\section{Discussion}

To ensure good quality in conducting FGs online, we provide some general advice to limit these potential limitations. Both online variants of FG are vulnerable to technical issues as well as human error. As briefly discussed, potential issues include participants having limited experience in using computers, IT or the particular communication software you are using. In order to limit such problems, our advice is to add some extra time for internet-based FG to deal with common technical problems. Organise the session to start 15 minutes early which would allow extra time to sort out technological hiccups and human uncertainties in using the technology (10). Secondly, try out the equipment beforehand. Thirdly, make sure you are able to inform participants whilst online on how to change the volume of their computer, how to mute and un-mute a microphone, etc.

In Nepal, one also has to be aware of cuts in electricity supply (so-called load-shedding) which is worse in certain parts of the country and certain times of the year. Check whether your participants have access to electricity, for example, through a generator or a battery for a mobile device.

For video-conference based online FGs, we advise you set appropriate ground rules. We always set ground rules for the running of an in-person FG, but for those conducted online, you may want to add a few specific rules related to those using audio only and those using video and audio. As with all types of FG, remind your participants this is a group discussion around certain specific questions or issues. Leave space for the development of new ideas and discussions, but at the same time, don't be shy in bringing the conversation back on focus. Sometimes a simple question is enough: "What does your comment have to do with the topic we are discussing today?" or "How does what you just said/wrote link to today's topic of xxxx?"

Don't be afraid to repeat (some of) your ground rules during the running of the FG if participants seem to have forgotten, get too animated, write insulting comments aimed at fellow participants, etc.

If possible, try to build up trust first between you as the facilitator and the participants beforehand. You may want to start by speaking to each participant by email or phone. Secondly, if possible, inform the participants of each other's names and perhaps job title. Whilst discussing sensitive topics, the facilitator can offer anonymity in online and audio-recorded FGs by concealing participants' identities. On the topic of 'abortion in rural Nepal' or 'HIV and pregnancy' participants could be allowed to log on under pseudonyms, not their own name, to create a safe research environment. This may attract participants who would never attend inperson FGs.

Finally, adding to the general confusion about size of groups in FG research $(7,9)$, we suggest you keep online video-recorded FG relatively small (3-6 participants). Having smaller groups ensures you do not lose your participants' attention; otherwise they may begin to check their emails, text friends, answer their mobile phones, etc. However, for online written FGs we would suggest a slightly larger sample (8 to 12 participants) as the method is less engaging and each individual participant is likely to contribute less than in a comparable traditional in-person FG or video-recorded internet-based FG.

As with all FGs, the role of the facilitator is very important. Internet-based FGs may require some additional skills, but they largely require the same as those used in conducting in-person FGs. These skills include knowledge of the topic as well as being able to facilitate a group discussion. They need to be able to listen, question and probe without directing the discussion (too much) and promote a group dynamic through, for example, encouraging less involved participants and reducing the risk of certain participants from dominating the FG discussion.

\section{Concluding remarks}

We would like to sum up by highlighting the following key issues for consideration:

1) Prepare the participants of audio/video FGs to arrive 15 minutes earlier to iron out any technological issues that could occur. Also try to have a second person present throughout the entire FG, so he or she can deal with technical issues whilst you facilitate the group discussion. 
2) Trust and rapport will still need to be developed through conversations prior to the FG.

3) Internet-based FGs could limit privacy and hence confidentiality. Participants sitting in shared offices, or at home could be interrupted by colleagues or family members during the FG. When using video these outsider may see or overhear other FG participants. 4) Body language and verbal context could be limited. This is true even when using video conferencing as there are many reasons why the video image of your participant can be poor. For example, you may see very little of the person if they sit too close to the camera, too far away from it, if the camera is out of focus, or if the lighting in the room is poor.

Taking into account the issues outlined in this paper, we think internet-based FGs, both written and audio/visual, can provide a cost-effective research method. It has potential in researching disperse populations which are difficult to reach such as some of the mountainous terrain of Nepal. The continuously developing technology makes online research more and more a viable option. Finally, a note of warning: all research methods have strengths and weaknesses. Of course, so do internet-based FGs. Therefore, researchers should be aware in developing their next study what particular strengths and weaknesses are likely to occur through using internet-based FG research.

\section{References}

1. Hollis V, Openshaw, S, Goble R. Conducting focus groups: purpose and practicalities. British Journal of Occupational Therapy 2002;65(1): p. 65-71.

2. van Teijlingen, ER,Pitchforth, E. Focus Group Research in Family Planning \& Reproductive Health Care. Journal of Family Planning \& Reproductive Health Care 2006;32(1): 30-32.

3. Barbour, R. Doing focus Groups. London: Sage.2007.

4. Krueger, RA, Casey, MA. Focus Groups (5th edn.). Thousand Oaks: Sage; 2015.

5. Liamputtong, P. Focus Group Methodology: Principle \& Practice. London: Sage; 2011.

6. van Teijlingen, E, Simkhada, P, Stephens, J. Doing focus groups in the health field: Some lessons from Nepal, Health Prospect2013; 12(1): 15-17. http://www.nepjol.info/index.php/HPROSPECT/article/view/8722/7111

7. Woodyatt,CR, Finneran, CA, Stephenson, R. In-Person Versus Online Focus Group Discussions: A Comparative Analysis of Data Quality. Qualitative Inquiry, 2016; 26(6): 741-749.

8. Murray, PJ. Using Virtual Focus Groups in Qualitative Research. Qualitative Health Research1997; 7(4):542-548.

9. Hurworth, R. Telephone Focus Groups Social Research Update 2004; issue 44 Guildford: University of Surrey (web site: http://sru. soc.surrey.ac.uk/SRU44.html ).

10. Tuttas, CA. Lessons Learned Using Web Conference Technology for Online Focus Group Interviews. Qualitative Health Research, 2015; 25(1):122-133. 